\title{
Timing of Antenatal Corticosteroid Administration in Monoamniotic Twins
}

\author{
Carolina Bibbo, MD ${ }^{1}$ Sarah R. Easter, MD ${ }^{1} \quad$ Michael Saadeh, BS ${ }^{1} \quad$ Sarah E. Little, MD, MPH \\ Julian N. Robinson, MD ${ }^{1}$
}

${ }^{1}$ Division of Maternal Fetal Medicine, Department of Obstetrics and Gynecology, Brigham and Women's Hospital, Boston, Massachusetts

Address for correspondence Carolina Bibbo, MD, Department of Obstetrics and Gynecology, Brigham and Women's Hospital, 75

Am J Perinatol Rep 2019;9:e153-e159.

Francis Street, Boston, MA 02115 (e-mail: cbibbo@partners.org).

\begin{abstract}
Keywords

- monoamniotic twins

- antenatal corticosteroids

- preterm delivery

- antenatal fetal testing

Objective This study was aimed to determine if different strategies of antenatal corticosteroid (ACS) administration in monoamniotic twins leads to receipt within 7 days of delivery.

Study Design This is a retrospective cohort of monoamniotic twins managed at a single institution from 2007 to 2017. Patients were classified as to whether ACS were administered upon admission or at a predetermined gestational age (grouped together as "routine") or for a change in clinical status ("indicated"). We used univariate analyses to associate ACS administration strategies with our primary outcome: receipt of ACS within 7 days of delivery. We then used generalized estimating equations to examine associations between fetal monitoring patterns and delivery within 1 week.

Results Twenty-four patients were included: eighteen patients in the "routine" group and six patients in the "indicated" group. There was no difference in optimal timing of ACS administration. Women experiencing delivery within the week were thrice more likely to spend on average more than 3 hours/day on the fetal monitor when compared with those who remained undelivered.

Conclusion Administration of ACS on admission is not effective. Fetal heart rate tracing surveillance might be a better methodology to predict delivery and guide ACS administration.
\end{abstract}

Monoamniotic twin pregnancies are rare, comprising $1 \%$ of all twins and $5 \%$ of monochorionic twins. ${ }^{1,2}$ Monoamniotic twins are uniquely associated with a significant risk of fetal death due to prolonged cord occlusion from cord entanglement as both twins share the same amniotic sac. This cord entanglement is almost inevitable and present in nearly all gestations., ${ }^{3,4}$ Literature, published more than 10 years ago, had reported a perinatal mortality rate as high as 30 to $70 \%^{5,6}$; however, more contemporary systematic reviews that have excluded anomalous fetuses and complications from twin-to-twin transfusion syndrome suggest a lower rate of 10 to $12 \% .^{7,8}$ Prenatal diagnosis, closer fetal surveillance, and elective preterm delivery have been attributed to this lower mortality rate. Prior

received

December 7, 2018

accepted after revision

December 11, 2018
DOI https://doi.org/

$10.1055 / s-0039-1687872$. ISSN 2157-6998. retrospective research supports that inpatient admission with frequent fetal testing is better at preventing intrauterine fetal death than outpatient management. ${ }^{5,9,10}$ However, there is still debate about the optimal antenatal management and delivery timing for these patients.

Given the significant risk of intrauterine death, elective premature delivery is guaranteed for all pregnancies with most data supporting cesarean delivery at 32 to 34 weeks. ${ }^{5,9,11}$ The administration of antenatal corticosteroids (ACS) between 23 and 34 weeks has shown to reduce the risk of neonatal death, respiratory distress syndrome, and neurological injury. ${ }^{12}$ Even though there is a paucity of research regarding the use of ACS in multiple pregnancies, administration is
Copyright $\odot 2019$ by Thieme Medical Publishers, Inc., 333 Seventh Avenue, New York, NY 10001, USA. Tel: +1(212) 584-4662.
License terms

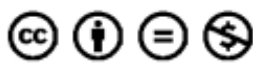


recommended regardless of fetal number. ${ }^{13,14}$ The original trial of Liggins and Howie demonstrated the need for ACS to be given within 1 week of delivery to be clinically efficacious. ${ }^{15}$ These findings have been corroborated by several trials. ${ }^{16,17}$

Optimal timing of ACS is particularly relevant in monoamniotic twins since their prematurity is inevitable. Prior studies have focused on neonatal outcomes with a wide variation on fetal testing strategies but the specific information regarding the rate of administration, protocol, and optimal timing of ACS is often lacking. $5,7,9,10$

We were interested to determine if different strategies for antenatal corticosteroid (ACS) administration in monoamniotic twin pregnancies would lead to optimal timing of ACS administration prior to delivery. Our hypothesis was that administration of ACS upon admission to the hospital or at a predetermined gestational age $(<30$ weeks) would not lead to receipt of steroids within 7 days of delivery. Furthermore, we wanted to demonstrate whether our cohort of inpatient monoamniotic twin management would elucidate any predictors for the timing of delivery.

\section{Materials and Methods}

This was a retrospective cohort study of monochorionic monoamniotic twin pregnancies at or beyond 24 weeks of gestational age that were managed at a single tertiary care center from 2007 to 2017. Potential cases of monoamniotic twin gestation were identified using hospital birth records. Inclusion criteria were a live monochorionic-monoamniotic twin gestation at time of admission, admission to the hospital between 24 and 28 weeks of gestation and delivery at our institution. Those patients who had a monoamniotic twin pregnancy due to iatrogenic or spontaneous intertwin membrane rupture were excluded. Conjoined twins were also excluded. The time of hospital admission was decided between the parents, obstetric provider, and neonatologists. Parents chose a gestational age at which they would want neonatal resuscitation if delivery was indicated.

All patients were admitted to the antepartum floor of our hospital where they were managed by the antepartum team which consisted of residents, fellows and a maternal-fetal medicine (MFM) specialist. As per hospital protocol, the antepartum fetal monitoring consisted of either twice (2007-2012) or thrice (2013-2017) daily fetal heart rate monitoring. The fetal heart rate monitoring consisted of 1 hour of fetal heart tracing each time the fetal heart rate was monitored (either twice or thrice daily). Additional monitoring was performed due to a finding on routine testing. If there were repetitive variable or late decelerations or the fetal heart tracing was difficult to interpret (discontinuous), the patient had prolonged fetal monitoring (the number of hours depending on the indication or concern of the obstetric provider). Repetitive decelerations were defined as more than two in a 30-minute period. Ultrasounds were performed weekly and fetal growth was estimated every 2 weeks. The degree of cord entanglement on ultrasound was noted but it was not an indication for additional fetal monitoring or delivery. Doppler's velocimetry studies were only preformed routinely when there were growth abnormalities and not to screen for umbilical cord compression. There was no protocol regarding timing of steroid administration, this was provider dependent.

Our primary exposure of interest was the strategy for the first course of ACS administration. We classified patients as to whether they received ACS on admission or at predetermined gestational age (grouped together as "routine"), or if ACS were reserved until a change in maternal or fetal clinical status ("indicated"). The change in clinical status was documented in the medical record. Our primary outcome of interest was receipt of ACS within 7 days of delivery. We made note of both first and repeat courses of ACS. Additional secondary outcomes of interest included rates of neonatal morbidity based on the work by Barrett and colleagues. ${ }^{18}$ We analyzed composite neonatal morbidity for the pregnancy overall and separately for presenting and nonpresenting twins. The composite neonatal outcome included one or more of the following: intubation for 2 or more days, birth trauma (including cephalohematoma, clavicular fracture, or long-bone fracture), infection requiring antibiotics, intraventricular hemorrhage (IVH), hypoxic ischemic encephalopathy, and death. We made note of need for intubation though this was excluded from our composite neonatal outcome if the duration of intubation was less than 2 days.

The associations between ACS administration strategy and primary and secondary outcomes were analyzed with Chisquare test or Fisher's exact test for categorical variables and Wilcoxon's rank sum test for continuous variables. Based on the results of our univariate analysis and our small sample size multivariate analysis was deferred. We next performed a secondary analysis to examine clinical factors associated with delivery within 1 week. For this analysis, we considered each week of patient hospitalization including the monitoring needs of each patient. We recorded daily hours on the fetal heart rate monitor for each woman throughout her inpatient hospitalization. Our outcome of interest for this secondary analysis was delivery within the week of interest. For this analysis, we first used univariate analysis to look for associations between clinical characteristics of interest including fetal monitoring patterns and delivery within the week. We then used generalized estimating equations to generate odds ratios examining the associations between fetal monitoring patterns and delivery while accounting for correlations in data over time. ${ }^{19}$

Demographic, obstetric, and labor and delivery data were collected by chart review. Study data were collected and managed using REDCap electronic data capture tools hosted by the institution. ${ }^{20}$ All analyses were performed with Statistical Analysis Software (SAS), Version 9.4 (Copyright 2013-2017, SAS Institute, Inc, Cary, NC). The study was approved by our Institutional Review Board (Protocol number 2016P002057, approved on 11/1/2016).

\section{Results}

During the study period of 2007 to 2017, 27 patients with a monochorionic-monoamniotic twin gestation delivered at our institution. Three patients were excluded; two of whom 


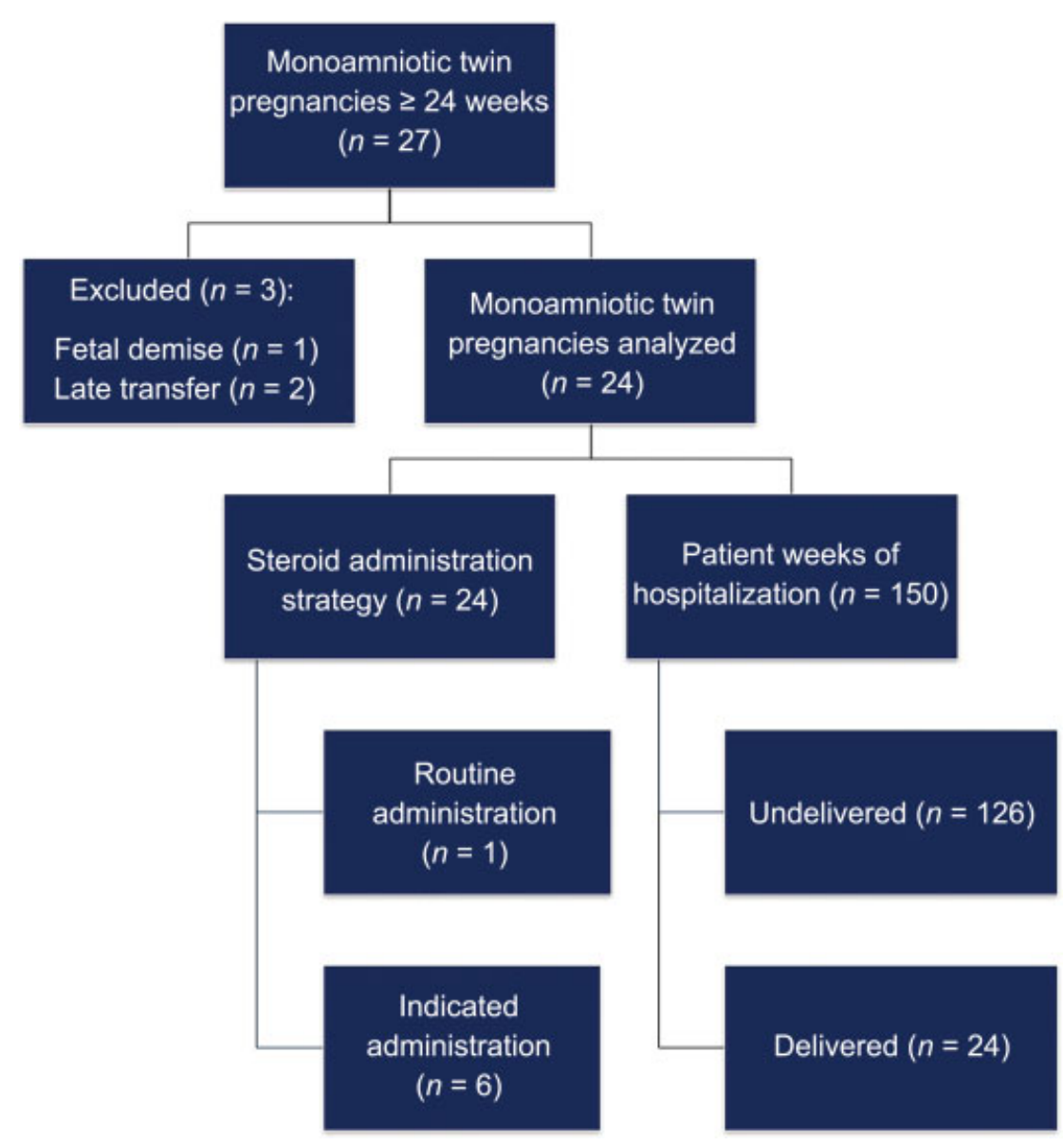

Fig. 1 Inclusion, classification and analysis of study population. This flow chart represents the inclusion criteria, classification pattern and analysis of our cohort of patients.

were transferred from outside hospitals for delivery and one as both twins had died at 25 weeks (prior to a planned admission at 26 weeks). - Fig. 1 shows the inclusion, classification, and analysis of patients. All patients in the cohort received a complete course of ACS. Eighteen patients received ACS upon admission (24-28 weeks) or at a predetermined gestational age (26-30 weeks), and six patients received ACS in the setting of a change in clinical status during the hospital admission. The change in clinical status $(n=6)$ encompassed four cases of fetal-heart rate tracing documented as nonreassuring that required prolonged monitoring, one new diagnosis of intrauterine growth restriction, and one case of preterm premature rupture of membranes. Seventy-nine percent $(19 / 24)$ of the patients received one complete repeat course of repeat ACS. The repeat course of ACS (only one course) was given due to nonreassuring fetal heart tracing $(n=7)$, concern for preterm labor $(n=1)$, or because the pregnancy was still undelivered after 30 weeks and there had been no recent ACS exposure $(n=11)$. There were no differences in maternal/obstetrics characteristics between these groups ( - Table 1 ). There were also no differences in neonatal outcomes (-Table 2 ).

The median gestational age at delivery of our cohort was 32.3 weeks (range: $28.1-35.3$ weeks). All patients in the cohort were delivered by cesarean section. The indications for delivery were scheduled (eight), nonreassuring fetal heart tracing
Table 1 Maternal and obstetric characteristics per antenatal corticosteroid administration strategy

\begin{tabular}{|l|l|l|l|}
\hline Characteristics & $\begin{array}{l}\text { Routine } \\
\text { ACS } \\
(\boldsymbol{n}=\mathbf{1 8})\end{array}$ & $\begin{array}{l}\text { Indicated } \\
\text { ACS } \\
(\boldsymbol{n}=6)\end{array}$ & $p$-Value \\
\hline Maternal age (y) & $32(28-38)$ & $35(31-41)$ & 0.40 \\
\hline Nulliparous & $9(50.0)$ & $2(33.3)$ & 0.64 \\
\hline $\begin{array}{l}\text { Spontaneous } \\
\text { conception }\end{array}$ & $14(77.8)$ & $4(66.7)$ & 0.62 \\
\hline $\begin{array}{l}\text { Intrauterine growth } \\
\text { restriction on } \\
\text { ultrasound }\end{array}$ & $5(27.8)$ & $2(33.3)$ & 1 \\
\hline $\begin{array}{l}\text { Cord entanglement } \\
\text { on ultrasound }\end{array}$ & $18(100.0)$ & $6(100.0)$ & 1 \\
\hline TTTS & 0 & 0 & 1 \\
\hline $\begin{array}{l}\text { Gestational age at } \\
\text { admission (wk) }\end{array}$ & $\begin{array}{l}26.5 \\
(25-28)\end{array}$ & $\begin{array}{l}27.3 \\
(26.3-27.7)\end{array}$ & 0.37 \\
\hline $\begin{array}{l}\text { Gestational age at } \\
\text { delivery (wk) }\end{array}$ & $\begin{array}{l}32.3 \\
(32.0-33.1)\end{array}$ & $\begin{array}{l}32.5 \\
(29.9-34)\end{array}$ & 0.95 \\
\hline ACS & $18(100.0)$ & $6(100.0)$ & 1 \\
\hline $\begin{array}{l}\text { Second course } \\
\text { of ACS }\end{array}$ & $16(88.9)$ & $3(50.0)$ & 0.08 \\
\hline
\end{tabular}

Abbreviations: ACS, antenatal corticosteroids; TTTS, twin-to-twin transfusion syndrome.

Note: Data are shown as $n(\%)$ or median (interquartile range). 
Table 2 Neonatal outcomes per antenatal corticosteroids administration strategy

\begin{tabular}{|c|c|c|c|}
\hline Outcome & $\begin{array}{l}\text { Routine ACS } \\
(n=36)\end{array}$ & $\begin{array}{l}\text { Indicated } \\
\text { ACS }(n=12)\end{array}$ & $p$-Value \\
\hline $\begin{array}{l}\text { NICU } \\
\text { admission }\end{array}$ & $36(100.0)$ & $12(100.0)$ & 1 \\
\hline $\begin{array}{l}\text { Birth weight } \\
\text { twin A (g) } \\
\text { Birth weight } \\
\text { twin B } \\
\text { (g) }\end{array}$ & $\begin{array}{l}1,757.5 \\
(1,587-1,927) \\
1,743 \\
(1,701-1,900)\end{array}$ & $\begin{array}{l}1,589 \\
(1,250-2,055) \\
1,666 \\
(1,285-1,920)\end{array}$ & $\begin{array}{l}0.48 \\
0.5\end{array}$ \\
\hline $\begin{array}{l}\text { Composite } \\
\text { twin } A^{a} \\
\text { Composite } \\
\text { twin } B^{a}\end{array}$ & $\begin{array}{l}5(27.8) \\
5(27.8)\end{array}$ & $\begin{array}{l}1(16.7) \\
1(16.7)\end{array}$ & $\begin{array}{l}1 \\
1\end{array}$ \\
\hline Stillbirth & 0 & 0 & $\mathrm{~N} / \mathrm{A}$ \\
\hline $\begin{array}{l}\text { Neonatal } \\
\text { death }\end{array}$ & 0 & $1(8.3)$ & 0.25 \\
\hline Fetal anomaly & $2(5.55)$ & $3(25)$ & 0.09 \\
\hline
\end{tabular}

Abbreviations: ACS, antenatal corticosteroids; N/A, not available; NICU, neonatal intensive care unit.

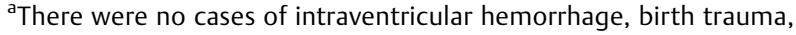
infection, hypoxic ischemic encephalopathy.

Note: Data are shown as $n$ (\%) or median (interquartile range).

status (13), preterm labor (two), and preeclampsia (one). There were no stillbirths in this cohort. All patients had cord entanglement reported on their ultrasounds. There was one neonatal demise at 2 days of life due to severe multiple congenital anomalies. This twin was born at 31.6 weeks with multicystic dysplastic kidneys, developed severe renal failure, and the parents opted to withdraw care. The mortality rate of our cohort was $2.1 \%$ (95\% confidence interval [CI]: 0.1 $11.1 \%$; $0 \%$ if anomalous fetuses were excluded). The other anomalies in the cohort were cardiac, renal, and pulmonary.

- Table 3 demonstrates that there was no difference in optimal timing of ACS administration (receipt within 7 days of delivery) regardless of the ACS administration strategy. None of the patients in either group received their first of course of ACS within 1 week of delivery. There was no difference between the groups in the rate of second course

Table 3 Administration of antenatal corticosteroids within 7 days of delivery per administration strategy

\begin{tabular}{|l|l|l|l|}
\hline Outcome & $\begin{array}{l}\text { Routine } \\
\text { ACS } \\
(\boldsymbol{n}=\mathbf{1 8})\end{array}$ & $\begin{array}{l}\text { Indicated } \\
\text { ACS } \\
(\boldsymbol{n}=6)\end{array}$ & $p$-Value \\
\hline $\begin{array}{l}\text { 1st course of ACS } \\
\text { within 7 d of delivery }\end{array}$ & 0 & 0 & 1 \\
\hline Repeat course of ACS & $16(88.9)$ & $4(66.7)$ & 0.25 \\
\hline $\begin{array}{l}\text { Repeat course of ACS } \\
\text { within 7 d of delivery }\end{array}$ & $10(62.5)$ & $2(50)$ & 1 \\
\hline $\begin{array}{l}\text { Any ACS within } \\
\text { 7 d of delivery }\end{array}$ & $10(55.6)$ & $2(33.3)$ & 0.64 \\
\hline
\end{tabular}

Abbreviation: ACS, antenatal corticosteroids.

Note: Data are shown as $n$ (\%) or median (interquartile range). of ACS administration within 7 days of delivery (55.6 vs. $33.3 \%, p=0.64$, - Table 3 ).

These 24 patients comprised 150 patient-week of inpatient observation. - Fig. 2 shows the pattern of fetal heart rate monitoring by week of gestation. This graph represents the median daily time that each patient spent on the monitor across gestation. Those patients who were delivered before 32 weeks spent more hours per week on the monitor (purple) while those who were delivered after 32 weeks spent fewer hours on the monitor (blue; - Fig. 2).

In the second analysis, women experiencing delivery within the week were more likely to spend more time on the fetal heart monitor compared with those who remained undelivered ( - Table 4 ). Those patients who spent on average more than 3 hours a day on the fetal heart monitor were thrice more likely to be delivered within the week ( - Table 4 ).

Twenty-five percent (6/24) of our patients delivered prior to 32 weeks. All of them had an objective change in clinical status that warranted prolonged fetal monitoring. Among these six patients, the least amount of time spent on a day on continuous fetal monitor was 5 hours and the most was 24 hours. In this group, the median time on the fetal heart rate monitor per day within 7 days prior to delivery was 12.5 hours (IQ 7.5-21.25 hour). Five of the six patients were delivered due to nonreassuring fetal heart tracing, and one was delivered for premature rupture of membranes/preterm labor. Among the six patients in the "indicated" ACS group, $50 \%$ (three/six) delivered before 32 weeks of gestation for nonreassuring fetal heart rate tracing and all three of them had a complete second course of ACS.

\section{Discussion}

Our study addresses the subject of timing of ACS administration in a population of monoamniotic twins which are certain to have a preterm delivery and in which antenatal ACS exposure would likely have clinical impact. Most of the published literature consists of small case series and systematic reviews that focus on neonatal outcomes based on antenatal fetal monitoring protocols. ${ }^{7,9,21,22}$ Little has been reported regarding the specific protocol for ACS administration, rate of ACS exposure, and optimal timing in these highrisk pregnancies. ${ }^{3,11,23}$ All patients in our study received ACS prior to delivery which is a higher rate than that reported in other international centers. ${ }^{11,23}$ Even though $100 \%$ of our patients received ACS, our study showed that neither routine administration upon hospital admission or predetermined gestational age nor administration based on subjective change in clinical status led to receipt of a first course of ACS within 7 days of delivery. Furthermore, even those patients who received the first course of ACS based on a clinical change of status did not deliver within 7 days. The rate of the second course of ACS administration within 7 days of delivery was higher in the routine ACS group (62.5\%) than the indicated ACS group (50\%); however, this difference was not significant. Our results showed that none of the strategies used in our hospital led to optimal administration of ACS. Upon chart review of our records, it became apparent 


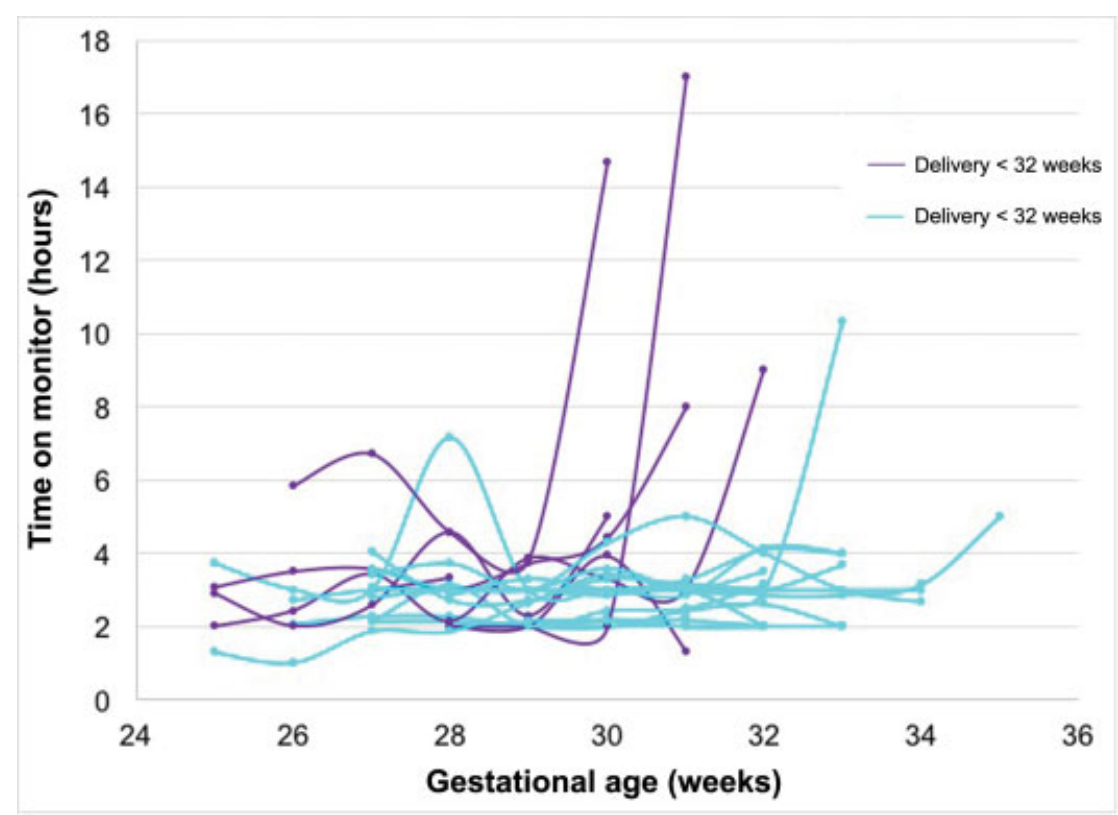

Fig. 2 Patterns of fetal monitoring by week of gestation. This graph represents the median daily time that each patient spent on the monitor across gestation. Those patients who were delivered before 32 weeks spent more hours per week on the monitor (purple) while those who were delivered after 32 weeks spent fewer hours on the monitor (blue).

Table 4 Fetal heart rate monitoring characteristics according to delivery within the week

\begin{tabular}{|l|l|l|l|}
\hline Characteristic & Undelivered $(\boldsymbol{n}=126)$ & Delivery within 7 days $(\boldsymbol{n}=\mathbf{2 4 )}$ & OR $(95 \% \mathrm{CI})$ \\
\hline Percentage change in h on monitor & $0(-0.02$ to 0.07$)$ & $0.06(-0.03$ to 0.70$)$ & $4.12(1.67-10.14)$ \\
\hline Increase in wkly h by 10\% & $29(23.0)$ & $12(50.0)$ & $3.34(1.56-7.19)$ \\
\hline Median daily h of monitoring & $3.0(2.14-3.14)$ & $3.2(2.0-5.0)$ & $1.52(1.19-1.94)$ \\
\hline Median $>3$ h of daily monitoring & $35(27.8)$ & $13(54.2)$ & $3.07(1.37-6.87)$ \\
\hline
\end{tabular}

Abbreviations: $\mathrm{Cl}$, confidence interval; OR, odds ratio.

Note: data are shown as $n(\%)$ or median (interquartile range).

that the change in clinical status was often related to instances where prolonged fetal heart rate monitor occurred but not always. Our analysis showed that patients who had been on the fetal monitor on average more than 3 hours a day were thrice more likely to be delivered within that week. This objective measurement, rather than just a subjective clinical change, is a novel clinical tool to better time ACS administration prior to delivery.

Whether umbilical cord occlusion can be anticipated is debatable. Given this unpredictability, most specialists recommend ACS administration upon admission. ${ }^{13,24}$ However, this very unpredictability and the potential fetal mortality associated with placental insufficiency and placental vascular anastomoses support a clinically driven approach. Our study suggests that changes in fetal heart rate patterns are associated with delivery within that week. Specifically, those patients who spent on average more than 3 hours a day on the fetal heart rate monitor were thrice more likely to be delivered that week. Even though we cannot conclude that intrauterine fetal death can be prevented with fetal surveillance, our results suggest that routine intermittent fetal surveillance leading to prolonged monitoring in a day is a harbinger of delivery within that week, giving a logical rationale for ACS administration.

The perceived importance of receiving ACS before delivery, combined with fear of missing the opportunity altogether, may overshadow the actual need for the medication to be given within 1 week of delivery to be efficacious. In our cohort, there were only six patients $(25 \%)$ who delivered prior to 32 weeks and all of them spent a prolonged time on fetal heart rate monitoring prior to delivery (median of 12.5 hours, range: 7.5-21.25 hours). From these results, one can assume that the need for prolonged fetal monitoring raises enough clinical concern that would allow for timely ACS administration prior to delivery. For those patients in the "indicated" ACS group, 50\% delivered before 32 weeks of gestation and all three of them had a complete second course of ACS. Although small numbers, the fact that no patients have missed administration ACS is reassuring as to the appropriateness of incorporating the fetal heart rate tracing interpretation into clinical management of ACS administration. By using this objective change in fetal monitoring pattern, we could increase the rate of optimal ACS administration and potentially improve neonatal outcomes. 
Of patients admitted for antenatal surveillance, we had no cases of stillbirths and only one neonatal demise from complications of multiple congenital anomalies. We decided to exclude cases of pregnancies affected by a stillbirth prior to admission ( $n=1)$ because our primary outcome was optimal timing of ACS administration and the focus of our analysis was not on neonatal morbidity or timing of delivery as in most published literature. The very low mortality rate of our institution ( 2.1 or $0 \%$ if anomalous fetuses were excluded) is consistent with prior research published by Prefumo et al and Baxi and Walsh., ${ }^{7,22}$ There is a wide variation in mortality rate reported in the literature with older data suggesting a mortality rate as high as 70\% which is often attributed to the fact those studies included demises due to fetal anomalies and fetal losses that were prior to 20 weeks. ${ }^{5,6}$

One of the strengths of our study is that even though it is a small case series, we gathered individual patient data regarding fetal heart rate monitoring. Our results provide novel data regarding an objective change in fetal heart rate monitoring that is associated with delivery within that week.

\section{Limitation}

Our study is not without limitations. Its retrospective nature being the largest. Another limitation is our small sample size and thus, we are likely underpowered to detect a difference in ACS administration strategies. Using the outcome of any ACS within 7 days of delivery, with rates of $55.6 \%$ in the routine group and 33.3\% in the indicated group, and an $\alpha$ of $5 \%$ a study would need 60 patients in each arm to detect a difference with $80 \%$ power. Given the rarity of monoamniotic twins and the underlying risk of stillbirth associated with these pregnancies, a randomized control trial of strategies for ACS administration would be extremely difficult to accomplish. Given the small sample size of our study, we cannot generalize our findings and a large-scale multicenter study is essential to corroborate our findings. Another limitation of our study is that our cohort of patients was established from hospital birth records. Historical records are never as accurate as prospective planned data collection.

\section{Conclusion}

In conclusion, our results demonstrate that ACS administration upon admission does not lead to optimal ACS administration. The use of inpatient fetal surveillance with the opportunity of prolonged fetal heart rate monitoring when clinically indicated might be a better methodology to predict delivery timing and likely better time ACS administration.

Note

The study was performed at Brigham and Women's Hospital, Boston, MA.

\section{Presentation}

These findings were presented as a poster at the 38th Annual Scientific Meeting of the Society for Maternal-Fetal Medicine in Dallas, TX; February 2018.

\section{Conflict of Interest}

The authors report no conflicts of interest and there was no financial support provided for this project.

\section{References}

1 Aisenbrey GA, Catanzarite VA, Hurley TJ, Spiegel JH, Schrimmer DB, Mendoza A. Monoamniotic and pseudomonoamniotic twins: sonographic diagnosis, detection of cord entanglement, and obstetric management. Obstet Gynecol 1995;86(02):218-222

2 Slotnick RN, Ortega JE. Monoamniotic twinning and zona manipulation: a survey of U.S. IVF centers correlating zona manipulation procedures and high-risk twinning frequency. J Assist Reprod Genet 1996;13(05):381-385

3 Pasquini L, Wimalasundera RC, Fichera A, Barigye O, Chappell L, Fisk NM. High perinatal survival in monoamniotic twins managed by prophylactic sulindac, intensive ultrasound surveillance, and Cesarean delivery at 32 weeks' gestation. Ultrasound Obstet Gynecol 2006;28(05):681-687

4 Dias T, Mahsud-Dornan S, Bhide A, Papageorghiou AT, Thilaganathan $B$. Cord entanglement and perinatal outcome in monoamniotic twin pregnancies. Ultrasound Obstet Gynecol 2010;35 (02):201-204

5 Ezra Y, Shveiky D, Ophir E, et al. Intensive management and early delivery reduce antenatal mortality in monoamniotic twin pregnancies. Acta Obstet Gynecol Scand 2005;84(05):432-435

6 Beasley E, Megerian G, Gerson A, Roberts NS. Monoamniotic twins: case series and proposal for antenatal management. Obstet Gynecol 1999;93(01):130-134

7 Prefumo F, Fichera A, Pagani G, Marella D, Valcamonico A, Frusca T. The natural history of monoamniotic twin pregnancies: a case series and systematic review of the literature. Prenat Diagn 2015; 35(03):274-280

8 Shub A, Walker SP. Planned early delivery versus expectant management for monoamniotic twins. Cochrane Database Syst Rev 2015;(04):CD008820

9 Heyborne KD, Porreco RP, Garite TJ, Phair K, Abril D; Obstetrix/ Pediatrix Research Study Group. Improved perinatal survival of monoamniotic twins with intensive inpatient monitoring. Am J Obstet Gynecol 2005;192(01):96-101

10 DeFalco LM, Sciscione AC, Megerian G, et al. Inpatient versus outpatient management of monoamniotic twins and outcomes. Am J Perinatol 2006;23(04):205-211

11 Van Mieghem T, De Heus R, Lewi L, et al. Prenatal management of monoamniotic twin pregnancies. Obstet Gynecol 2014;124(03): 498-506

12 Melamed N, Shah J, Yoon EW, et al; Canadian Neonatal Network Investigators. The role of antenatal corticosteroids in twin pregnancies complicated by preterm birth. Am J Obstet Gynecol 2016; 215(04):482.e1-482.e9

13 American College of Obstetricians and Gynecologists; Society for Maternal-Fetal Medicine. ACOG Practice Bulletin No. 144: Multifetal gestations: twin, triplet, and higher-order multifetal pregnancies. Obstet Gynecol 2014;123(05):1118-1132

14 Roberts D, Brown J, Medley N, Dalziel SR. Antenatal corticosteroids for accelerating fetal lung maturation for women at risk of preterm birth. Cochrane Database Syst Rev 2017;3:CD004454

15 Liggins GC, Howie RN. A controlled trial of antepartum glucocorticoid treatment for prevention of the respiratory distress syndrome in premature infants. Pediatrics 1972;50(04):515-525

16 Roberts D, Dalziel S. Antenatal corticosteroids for accelerating fetal lung maturation for women at risk of preterm birth. Cochrane Database Syst Rev 2006;(03):CD004454

17 Palas D, Ehlinger V, Alberge C, et al. Efficacy of antenatal corticosteroids in preterm twins: the EPIPAGE-2 cohort study. BJOG 2018;125(09):1164-1170 
18 Barrett JF, Hannah ME, Hutton EK, et al; Twin Birth Study Collaborative Group. A randomized trial of planned cesarean or vaginal delivery for twin pregnancy. N Engl J Med 2013;369(14): 1295-1305

19 Zeger SL, Liang KY, Albert PS. Models for longitudinal data: a generalized estimating equation approach. Biometrics 1988;44 (04):1049-1060

20 Harris PA, Taylor R, Thielke R, Payne J, Gonzalez N, Conde JG. Research electronic data capture (REDCap)-a metadata-driven methodology and workflow process for providing translational research informatics support. J Biomed Inform 2009;42(02):377-381
21 Murata M, Ishii K, Kamitomo M, et al. Perinatal outcome and clinical features of monochorionic monoamniotic twin gestation. J Obstet Gynaecol Res 2013;39(05):922-925

22 Baxi LV, Walsh CA. Monoamniotic twins in contemporary practice: a single-center study of perinatal outcomes. J Matern Fetal Neonatal Med 2010;23(06):506-510

23 Hack KE, Derks JB, Schaap AH, et al. Perinatal outcome of monoamniotic twin pregnancies. Obstet Gynecol 2009;113 (2 Pt 1):353-360

24 Post A, Heyborne K. Managing monoamniotic twin pregnancies. Clin Obstet Gynecol 2015;58(03):643-653 